\title{
Novel grid combined with peripheral distortion correction for ultra-widefield image grading of age-related macular degeneration
}

This article was published in the following Dove Press journal:

Clinical Ophthalmology

8 November 2017

Number of times this article has been viewed

\author{
Patrick Oellers ${ }^{1, *}$ \\ Inês Laíns ${ }^{1,2, *}$ \\ Steven Mach' \\ Shady Garas' \\ Ivana K Kim' \\ Demetrios G Vavvas' \\ Joan W Miller' \\ Deeba Husain' \\ John B Miller'
}

'Retina Service, Department of Ophthalmology, Massachusetts Eye and Ear, Harvard Medical School, Boston, MA, USA; ${ }^{2}$ Faculty of Medicine, University of Coimbra, Coimbra, Portugal

*These authors contributed equally to this work
Correspondence: John B Miller Department of Ophthalmology, Retina Service, Massachusetts Eye and Ear, Harvard Medical School, 243 Charles Street, Boston, MA 02। I4, USA

Tel +l 6175733750

Fax + I 6175733698

Email john_miller@meei.harvard.edu
Purpose: Eyes with age-related macular degeneration (AMD) often harbor pathological changes in the retinal periphery and perimacular region. These extramacular changes have not been well classified, but may be phenotypically and functionally relevant. The purpose of this study was to demonstrate a novel grid to systematically study peripheral retinal abnormalities in AMD using geometric distortion-corrected ultra-widefield (UWF) imaging.

Methods: This is a cross-sectional observational case series. Consecutive patients with AMD without any other coexisting vitreoretinal disease and control patients over age 50 without AMD or any other vitreoretinal disease were imaged using Optos 200 Tx. Captured $200^{\circ}$ UWF images were corrected for peripheral geometric distortion using Optos transformation software. A newly developed grid to study perimacular and peripheral abnormalities in AMD was then projected onto the images.

Results: Peripheral and perimacular changes such as drusen, retinal pigment epithelium changes and atrophy were found in patients with AMD. The presented grid in conjunction with geometric distortion-corrected UWF images allowed for systematic study of these peripheral changes in AMD.

Conclusion: We present a novel grid to study peripheral and posterior pole changes in AMD. The grid is unique in that it adds a perimacular zone, which may be important in characterizing certain phenotypes in AMD. Our UWF images were corrected for geometric peripheral distortion to accurately reflect the anatomical dimensions of the retina. This grid offers a reliable and reproducible foundation for the exploration of peripheral retinal pathology associated with AMD.

Keywords: ultra-widefield, autofluorescence, macular degeneration, grid, periphery, drusen, retinal pigment epithelium changes

\section{Introduction}

Age-related macular degeneration (AMD) is a leading cause of blindness in developed countries. Systematic study and grading of AMD has been limited to the posterior pole until recently. ${ }^{1,2}$ However, it is well recognized that pathological changes, such as drusen and retinal pigment epithelium (RPE) changes, also exist in the peripheral fundus in patients with AMD. ${ }^{3-8}$ In contrast to the progress that has been made on macular-derived findings of this disease, the impact on function and disease progression of peripheral retinal changes remains not well understood. While regular fundus photography, as well as optical coherence tomography, can be used to objectively document and study macular changes, systematic investigation of the peripheral fundus has been more challenging. ${ }^{1,9}$ 
Recently, ultra-widefield (UWF) fundus imaging has been established to create high-resolution images of the peripheral and central retina, which can be used for grading of peripheral retinal diseases. This has been widely performed for diabetic retinopathy and more recently for AMD..$^{10,11}$ In fact, UWF imaging has already demonstrated that peripheral abnormalities seen on pseudocolor and autofluorescence imaging are more prevalent in AMD than healthy patients. ${ }^{3,12}$

These peripheral abnormalities in AMD may be genetically and phenotypically distinct and hence need to be studied. For example, one recent study that used clinical examination alone without standardized imaging suggests that peripheral drusen and reticular pigment changes are associated with AMD severity. Further, peripheral drusen were associated with $\mathrm{CFHY} 402 \mathrm{H}$ genotype and reticular pigment changes were associated with CFHrs 1410996 genotype. ${ }^{6}$ Peripheral disease may also impact function in AMD. Impairment in dark adaptation, a rod-driven process and hence topographically mapped to the peripheral retina, has been found to be affected in early-stage macular degeneration as well as to be correlated with AMD disease severity. ${ }^{13,14}$ To this end, systematic UWF imaging grid grading systems are needed to methodically study peripheral abnormalities in AMD.

While different groups work on refinement of grids that can be used for grading of peripheral abnormalities, one of the difficulties encountered in the systematic grading of UWF images has been that these images are distorted in the periphery and hence do not reflect the actual dimensions of the retina. ${ }^{15}$

We present a novel UWF imaging-based grid grading system that incorporates correction for peripheral image distortion and defines regions of the periphery, providing a foundation for ongoing cohort studies of patients with AMD.

\section{Methods}

This study is part of a prospective, cross-sectional, observational project on AMD biomarkers. It was conducted in accordance with HIPAA (Health Insurance Portability and Accountability Act) requirements and the tenets of the Declaration of Helsinki, and was approved by the Massachusetts Eye and Ear (MEE) Institutional Review Board. All included participants provided written informed consent.

\section{Inclusion and exclusion criteria}

We recruited and consented consecutive patients with a diagnosis of AMD when they visited the Retina Service of MEE during their regular appointments. We excluded subjects with any other vitreoretinal disease, active uveitis or ocular infection, significant media opacities that precluded the observation of the ocular fundus, refractive error equal to or greater than $6 \mathrm{D}$ of spherical equivalent, past history of retinal surgery, history of any ocular surgery or intraocular procedure (such as laser and intraocular injections) within the last 90 days prior to enrollment and diagnosis of diabetes mellitus, with or without concomitant diabetic retinopathy. Additionally, a control group of subjects aged 50 years or older, without any evidence of AMD in both eyes, was included, and the same exclusion criteria were applied.

\section{Image acquisition}

All participants were imaged using the Optos 200 Tx camera in three gaze positions (Optos Inc, Dunfermline, UK), both with pseudocolor and autofluorescence. The central image taken in primary gaze missed some aspects of the retinal periphery. The two additional images taken in upgaze and downgaze covered these additional areas, as shown in Figure 1.

\section{Image processing}

Following acquisition, images underwent transformation using Optos stereographic projection software (ProView), which takes into account the optical imaging system and the ocular geometry to map each pixel to a consistent, spherical geometry. This preserves angular features and enables accurate representation of retinal features throughout the entire image. This software is incorporated into the newer Optos California device and available as an upgrade for both Optos Advance and V2 Advantage software for use with Optos 200 Tx.

The resulting images are $33.86 \times 33.86 \mathrm{~cm}$ in size with a resolution of 300 dots per inch. Thereafter, a novel fundus grid, specifically created to help grade central and peripheral AMD, was applied onto each of the six created images. No image montage was created. The grid consists of three concentric circles and crosshairs centered on the fovea.

The first circle surrounds the macula within the vascular arcades, with a radius of $29 \mathrm{~mm}$. This circle is based on the Early Treatment of Diabetic Retinopathy Study (ETDRS) grid used by the Age-related Eye Disease Study (AREDS). ${ }^{1}$ The ETDRS grid was first developed to aid in localization of diabetic macular edema and the assessment of diabetic retinopathy, ${ }^{16}$ but it also provides a reliable means for the assessment of central drusen burden in AMD.

The second circle encompasses the perimacular area, including both temporal arcades, with a radius of $65 \mathrm{~mm}$. Through clinical experience, we have found that many 

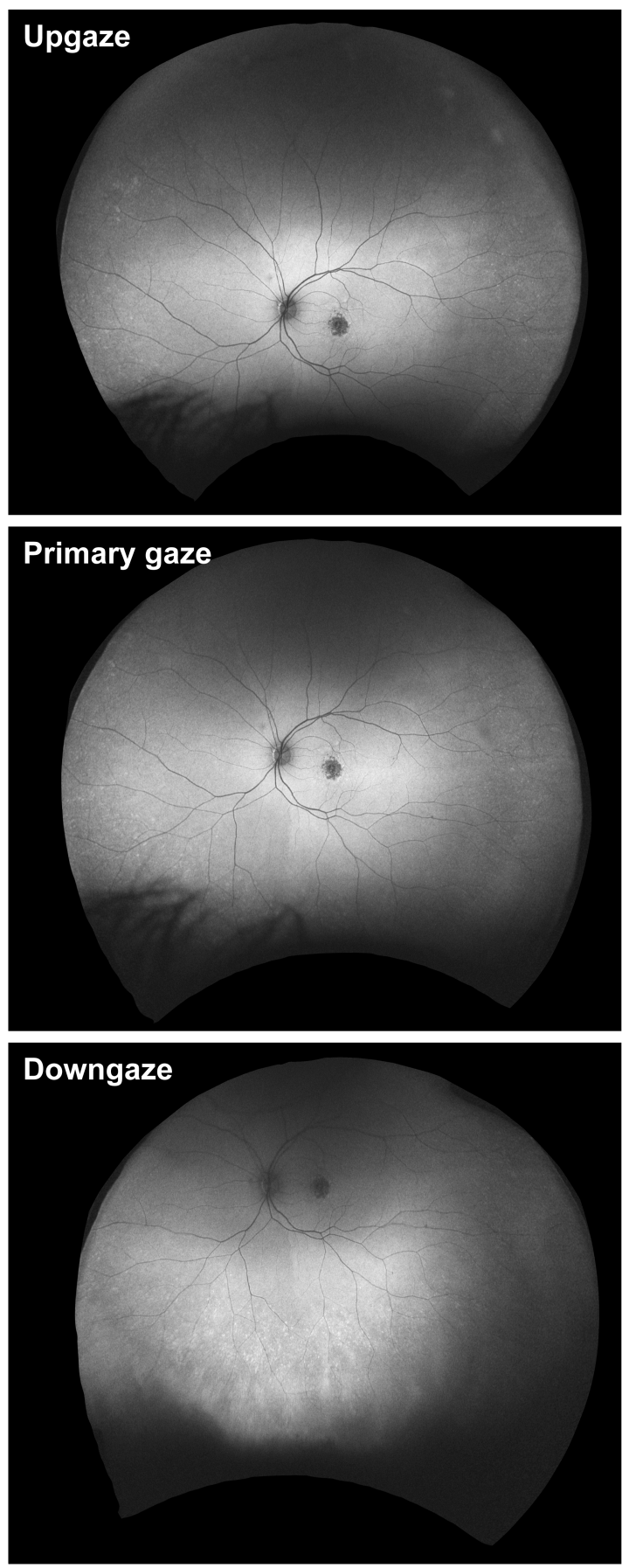

Figure I Gaze positions to optimally capture entire fundus in ultra-widefield imaging. Images were routinely captured in primary gaze, upgaze and downgaze. While primary gaze images reflect the temporal and nasal periphery reasonably well, it is necessary to capture images in upgaze and downgaze to better show the superior and inferior periphery as demonstrated in this ultra-widefield autofluorescence image sequence of a left eye.

patients have a high proportion of pathology in this posterior location, yet one that is outside the macula. By including this pericentral location and not just the mid- and far periphery outside the macula, we hope to better categorize these clinical subtypes.

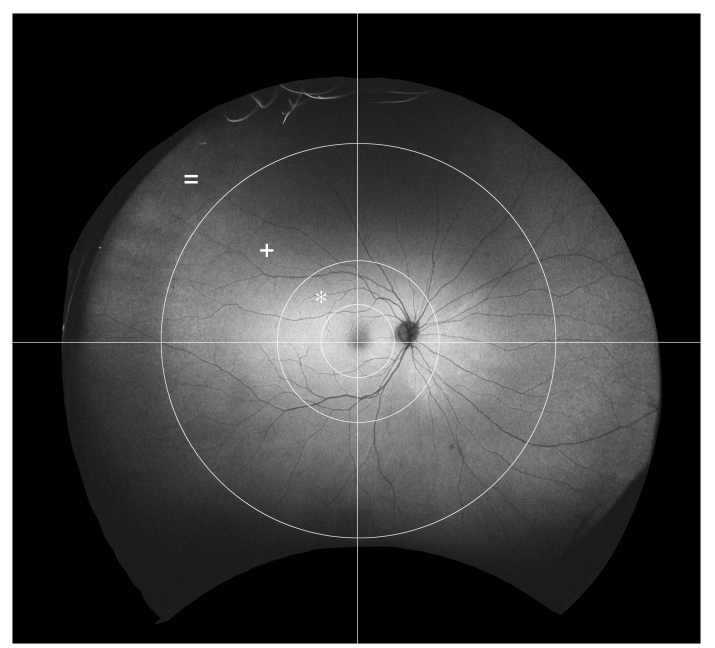

Figure 2 Novel grid to systematically grade peripheral abnormalities in age-related macular degeneration. The grid consists of three concentric circles and two lines. The central circle surrounds the macula area. The middle circle surrounds the perimacular area $(*)$. The largest circle separates the mid periphery $(+)$ from the far periphery $(=)$. A vertical line through the fovea separates temporal from nasal quadrants. A horizontal line through the fovea separates superior from inferior quadrants.

The last and most peripheral circle separates the midperipheral area from the far periphery. This circle is drawn with a radius of $160 \mathrm{~mm}$, which was chosen based on the location of the vortex veins. With this grid, the extramacular retina can be divided into 12 zones with the perimacular area, mid periphery and far periphery in each of the four quadrants: superotemporal, superonasal, inferonasal and inferotemporal (Figure 2).

The circles and the crosshairs were all centered on the fovea as opposed to the optic nerve. By centering on the fovea, the perimacular area can be properly positioned with a fixed radius. It also allows the nasal mid periphery to be more analogous to the temporal mid periphery than if the zones were centered on the optic nerve, in which case the macula would be included in the temporal mid-peripheral zones.

Most importantly, the grid corrects for the peripheral distortion inherent to the image acquisition of this UWF imaging device. This allows for an accurate measurement throughout the periphery.

As a result of the image processing, the optic disk measures about $10 \mathrm{~mm}$ in these images, whereas it is approximately $1.5 \mathrm{~mm}$ in the average human fundus. In this way, images project to about 6.7 times the size of the actual fundus. Images were then stored digitally and used for analysis of macular and peripheral pathology.

\section{Results \\ Case presentations}

To demonstrate feasibility of fundus grading with our novel grid projected onto distortion-corrected UWF imaging we 
detail findings of two patients with AMD and one control patient without AMD.

\section{Case I}

A 69-year-old male presented with AMD in both eyes. Bestcorrected visual acuity (BCVA) was 20/40 in the right eye and $20 / 25$ in the left eye. In the right eye (Figure 3), there were central RPE changes and macular drusen (Figure 3A-C). There was some RPE atrophy demonstrated by perifoveal hypoautofluorescence (Figure 3D-F). The perimacular zone demonstrated extensive drusen, most prominent inferotemporally and more moderate superotemporally and superonasally (Figure $3 \mathrm{~A}$ and $\mathrm{C}$ ), with very few drusen present inferonasally (Figure 3C and F). These drusen extended into the mid periphery and were best seen in upgaze (Figure 3A and D). A few loose drusen were also present in mid periphery inferonasally and inferotemporally, best seen in downgaze (Figure $3 \mathrm{C}$ and F). The nasal far periphery also harbored drusen, best seen in upgaze for the superonasal quadrant (Figure $3 \mathrm{~A}$ and $\mathrm{E}$ ) and in downgaze for the inferonasal quadrant (Figure 3C and $\mathrm{F}$ ). There were isolated drusen in the far periphery superotemporally (Figure 3A), but no pathological abnormalities were seen inferotemporally (Figure 3C and F).

\section{Case 2}

A 62-year-old female presented with geographic atrophy and drusen in both eyes. BCVA was 20/50 in the right eye and 20/125 in the left eye. The right eye (Figure 4) demonstrated juxtafoveal geographic atrophy as well as several drusen (Figure 4A-C). Few drusen extended into the temporal perimacular area (Figure 4). There was a cluster of nasal and superonasal drusen in the far periphery (Figure 4A, B, $\mathrm{D}$ and $\mathrm{E}$ ). Few drusen were present in the superotemporal and inferotemporal mid- and far periphery, best seen on autofluorescence images (Figure 4D-F). The inferior fundus had several drusen, mostly along the border of the mid- and far periphery (Figure 4C-F). In addition, there were relatively focal hyperautofluorescent and hypoautofluorescent changes in the mid-peripheral nasal to the optic disk, representing RPE atrophy with adjacent increased RPE metabolism. The patchy color changes in the temporal far periphery on pseudocolor images (Figure 4A and B) were artifacts and had no correlation on respective autofluorescence images (Figure 4D and E).

\section{Case 3}

A 60-year-old female presented with recurrent corneal abrasions, without signs of AMD or any other vitreoretinal disease.
BCVA was 20/25 in the right eye and 20/20 in the left eye. In the right eye, the macula appeared normal without any drusen or RPE atrophy (Figure 5). There was focal RPE atrophy in the superonasal quadrant of the mid periphery (Figure 5A and D). There were nonspecific patchy hyperautofluorescent changes in the inferotemporal quadrant of the mid- and far periphery (Figure 5F). Speckled dots in the superior fundus were seen on pseudocolor images (Figure 5A) representing artifacts instead of drusen, and there was no correlation on autofluorescence imaging (Figure 5D).

\section{Discussion}

We here present a novel UWF imaging grid grading system that can be used for systematic studies of perimacular and peripheral lesions in AMD. Our grading technique has several advantages over other previously described systems, by offering distortion correction to UWF imaging as well as a more refined grid that adds a perimacular zone next to central, mid-peripheral and far-peripheral zones.

Since its inception, UWF imaging has been used to study peripheral findings in AMD. Csutak et al reported a high rate of agreement between regular $45^{\circ}$ central fundus photography and UWF imaging for grading of abnormalities in the macula. The authors demonstrated that the level of detail and resolution of UWF imaging should be sufficient to grade findings in the macular region as well, likely allowing comparison of UWF imaging studies with studies that were based on conventional fundus photography. ${ }^{17}$

In 2015, Lengyel et al introduced the first grading system for peripheral lesions in AMD based on UWF imaging. The grid suggested by these authors consists of three concentric rings representing the fovea, perifovea and macular zones analogous with the ETDRS grid, in addition to a fourth zone for the mid periphery and fifth zone for the far periphery. While zones 1-3 were chosen based on prespecified parameters of the International AMD Classification Study and hence allow comparison with prior studies, peripheral zones 4 and 5 were chosen arbitrarily. The study demonstrated feasibility of grading peripheral lesions (namely RPE changes, drusen, mixed lesion and atrophy) as well as macular abnormalities in patients with AMD using autofluorescence and pseudocolor UWF imaging with high intergrader agreement. ${ }^{10}$ While this study certainly added to our understanding of peripheral AMD findings, it is limited by not correcting for the distortion of images in the periphery.

Our novel method offers several theoretical advantages by reducing distortion of the images of the retinal periphery and adding an additional perimacular zone for grading. Optos 

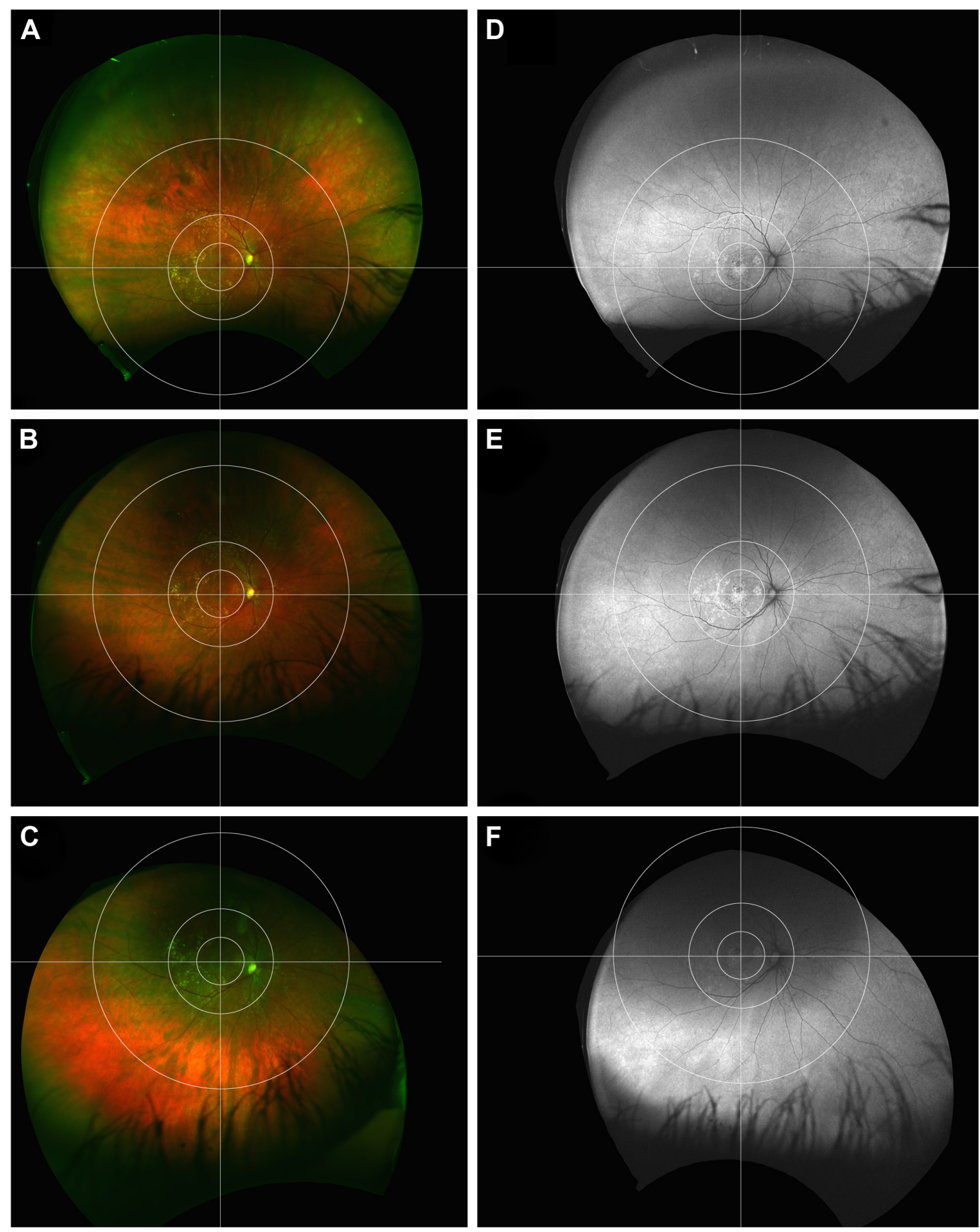

Figure 3 Patient with age-related macular degeneration and widespread perimacular and peripheral abnormalities. Ultra-widefield pseudocolor images of the right eye demonstrate foveal retinal pigment epithelium (RPE) changes along with macular drusen in all quadrants. There are extensive drusenoid changes in the perimacular area in the temporal quadrants, as well as mid-peripheral and far-peripheral drusen and RPE changes (A-C). Analogous to this, ultra-widefield autofluorescence shows mixed hyper/ hypoautofluorescence in the macula and hyperautofluorescence in the perimacula. The mid- and far periphery show partially mottled hyperautofluorescence (D-F). Upgaze (A and $\mathbf{D})$; primary gaze (B and $\mathbf{E})$, downgaze ( $\mathbf{C}$ and $\mathbf{F})$.

captures images of the far-peripheral temporal and nasal retina by utilizing an ellipsoid mirror, which leads to significant distortion of the peripheral image. When analyzing these distorted images, grading may be prone to artifacts as well as over- or underestimation of observed abnormalities.
In addition, unless the degree of image distortion would be stable in future imaging machines, image analysis may not be reproducible.

To reflect this, we used a novel image transformation software developed by Optos that preserves the spherical 

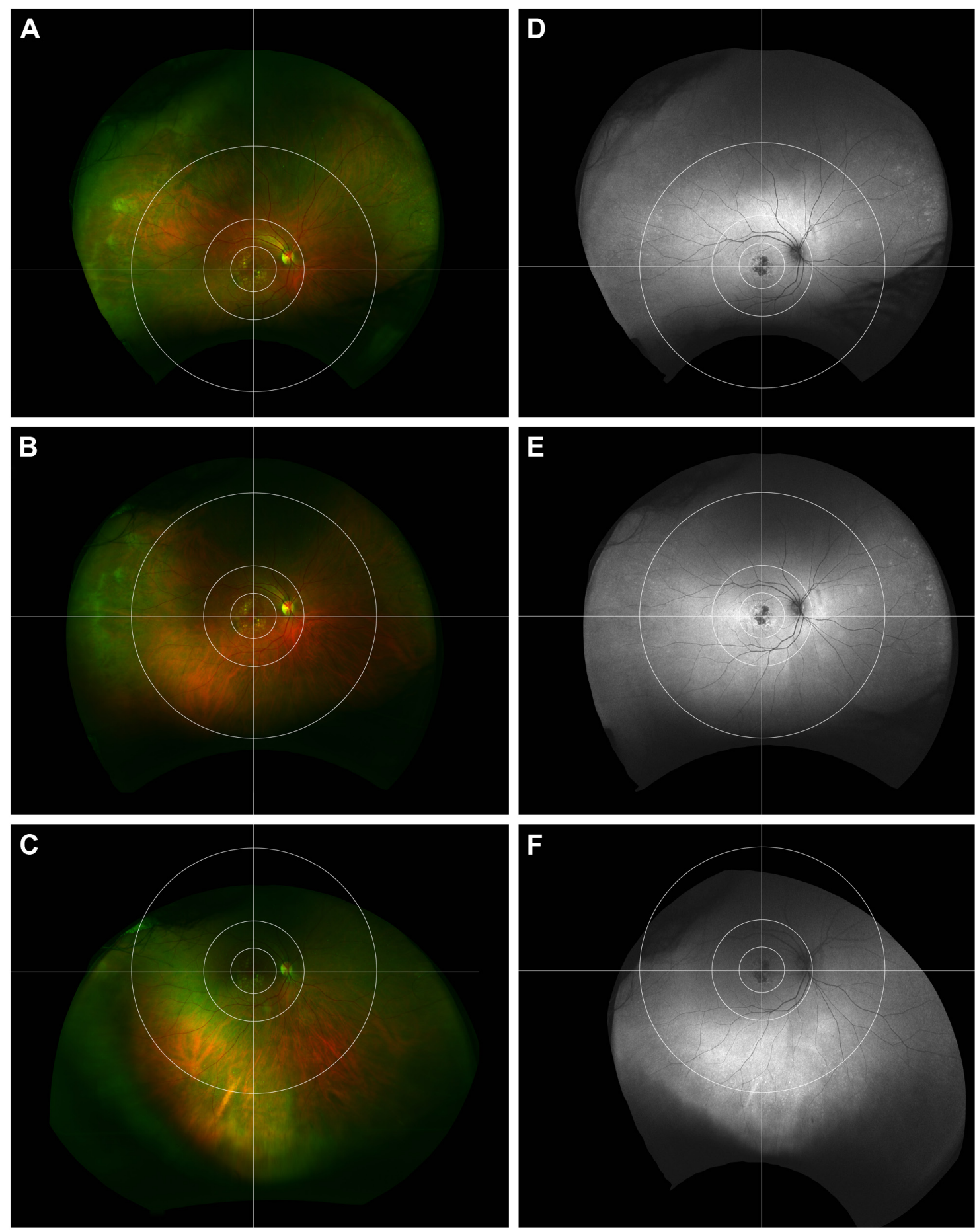

Figure 4 Patient with age-related macular degeneration and peripheral abnormalities. Ultra-widefield pseudocolor images of the left eye demonstrate juxtafoveal geographic atrophy and macular drusen, partially extending into the perimacular region. There are a few drusen, mostly in the nasal far periphery, but also inferior along the border of mid- and far periphery $(\mathbf{A}-\mathbf{C})$. Corresponding autofluorescence images demonstrate brisk hypoautofluorescence juxtafoveally with adjacent hyperautofluorescence. There is hyperautofluorescence, which extends into the temporal perimacular as well as speckled hyperautofluorescence, mostly nasally and inferiorly (D-F). Upgaze (A and D); primary gaze (B and $\mathbf{E})$, downgaze ( $\mathbf{C}$ and $\mathbf{F})$.

geometry of the peripheral retina in UWF imaging, which hopefully should pave the way for adequate, reliable and reproducible investigation of peripheral abnormalities, especially future quantitative analyses. Furthermore, image distortion correction is now commercially available and integrated into newer-generation devices. Also, the grid suggested by Lengyel et al as well as the AREDS group separates the peripheral retina into the far- and mid-peripheral zone, whereas we have chosen an additional zone for the perimacular area just outside the vascular arcades. We 

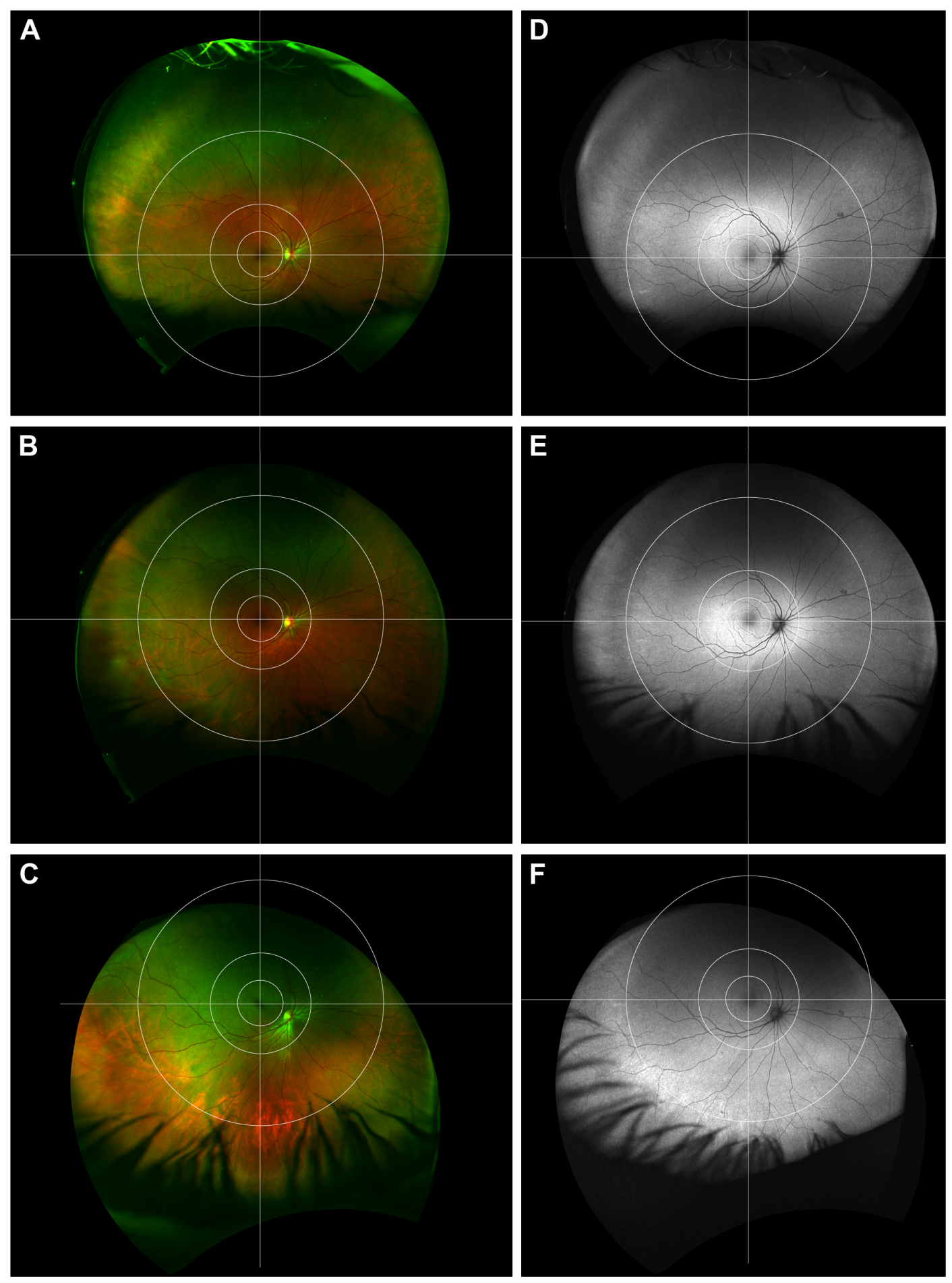

Figure 5 Patient without age-related macular degeneration and nonspecific peripheral retinal changes. Ultra-widefield pseudocolor images of the right eye demonstrate normal macula and a few nonspecific changes in the peripheral retina (A-C). Autofluorescence imaging demonstrates focal retinal pigment epithelium atrophy in the superonasal mid periphery as well as patchy hyperautofluorescence inferotemporally along the border of mid- and far periphery (D-F). Upgaze (A and D); primary gaze (B and $\mathbf{E})$, downgaze ( $\mathbf{C}$ and $\mathbf{F})$.

feel that the perimacular zone may be important in future classification of peripheral AMD. In our clinical experience, there are many patients with peripheral drusen and RPE abnormalities solely in the perimacular area without findings in the far-peripheral retina, which is exemplified by patient
1 in this series. Hypothesizing that this may be a specific phenotype, we believe there is value by adding a specific zone for grading the perimacular area.

Our new method has some limitations for grading of peripheral pathology. Optos creates pseudocolor images by 
way of laser scanning ophthalmoscopy, which in theory is prone to artifacts or misinterpretation of findings. Most retina specialists accept this limitation because conventional fundus photography is more time intensive and limited to the mid periphery. Ultra-widefield autofluorescence imaging can provide useful information, but should be interpreted in conjunction with pseudocolor images to avoid misinterpretation of potential artifacts. Our grid is limited to patients with up to $-6 \mathrm{D}$ refractive error due to the enlarged fundus dimensions associated with myopia. While the here-described methodology system is relatively new, we suggest that with further research, UWF imaging in conjunction with image grading methodologies such as described here has potential to assist in teleophthalmology system networks in the future. ${ }^{18}$

In summary, we present a novel grid applied to peripheral geometric distortion correction UWF imaging. The grid allows analysis of the peripheral retinal fundus divided into 12 reproducible zones. This systematic assessment will be used in future clinical studies to unravel the peripheral phenotypes and associations of AMD.

\section{Acknowledgment}

The authors would like to thank Optos (Optos Inc, Dunfermline, UK) for technical assistance and help with the manuscript.

\section{Disclosure}

The authors report no conflicts of interest in this work.

\section{References}

1. Age-Related Eye Disease Study Research Group. The Age-Related Eye Disease Study system for classifying age-related macular degeneration from stereoscopic color fundus photographs: the Age-Related Eye Disease Study Report Number 6. Am J Ophthalmol. 2001;132(5): 668-681.

2. Bird AC, Bressler NM, Bressler SB, et al. An international classification and grading system for age-related maculopathy and age-related macular degeneration. The International ARM Epidemiological Study Group. Surv Ophthalmol. 1995;39(5):367-374.

3. Heussen FM, Tan CS, Sadda SR. Prevalence of peripheral abnormalities on ultra-widefield greenlight $(532 \mathrm{~nm})$ autofluorescence imaging at a tertiary care center. Invest Ophthalmol Vis Sci. 2012;53(10):6526-6531.

4. Reznicek L, Wasfy T, StumpfC, et al. Peripheral fundus autofluorescence is increased in age-related macular degeneration. Invest Ophthalmol Vis Sci. 2012;53(4):2193-2198.

\section{Clinical Ophthalmology}

\section{Publish your work in this journal}

Clinical Ophthalmology is an international, peer-reviewed journal covering all subspecialties within ophthalmology. Key topics include: Optometry; Visual science; Pharmacology and drug therapy in eye diseases; Basic Sciences; Primary and Secondary eye care; Patient Safety and Quality of Care Improvements. This journal is indexed on Submit your manuscript here: http://www.dovepress.com/clinical-ophthalmology-journal
5. Rudolf M, Clark ME, Chimento MF, Li CM, Medeiros NE, Curcio CA. Prevalence and morphology of druse types in the macula and periphery of eyes with age-related maculopathy. Invest Ophthalmol Vis Sci. 2008;49(3):1200-1209.

6. Seddon JM, Reynolds R, Rosner B. Peripheral retinal drusen and reticular pigment: association with CFHY402H and CFHrs1410996 genotypes in family and twin studies. Invest Ophthalmol Vis Sci. 2009;50(2):586-591.

7. Tan CS, Heussen F, Sadda SR. Peripheral autofluorescence and clinical findings in neovascular and non-neovascular age-related macular degeneration. Ophthalmology. 2013;120(6):1271-1277.

8. Witmer MT, Kozbial A, Daniel S, Kiss S. Peripheral autofluorescence findings in age-related macular degeneration. Acta Ophthalmol. 2012; 90(6): e428-e433.

9. Kanagasingam Y, Bhuiyan A, Abràmoff MD, Smith RT, Goldschmidt L, Wong TY. Progress on retinal image analysis for age related macular degeneration. Prog Retin Eye Res. 2014;38:20-42.

10. Lengyel I, Csutak A, Florea D, et al. A population-based ultra-widefield digital image grading study for age-related macular degenerationlike lesions at the peripheral retina. Ophthalmology. 2015;122(7): $1340-1347$.

11. Soliman AZ, Silva PS, Aiello LP, Sun JK. Ultra-wide field retinal imaging in detection, classification, and management of diabetic retinopathy. Semin Ophthalmol. 2012;27(5-6):221-227.

12. Writing Committee for the OPTOS PEripheral RetinA (OPERA) study (Ancillary Study of Age-Related Eye Disease Study 2), Domalpally A, Clemons TE, Danis RP, et al. Peripheral retinal changes associated with age-related macular degeneration in the Age-Related Eye Disease Study 2: Age-Related Eye Disease Study 2 report number 12 by the Age-Related Eye Disease Study 2 Optos PEripheral RetinA (OPERA) Study Research Group. Ophthalmology. 2017;124(4): 479-487.

13. Flamendorf J, Agrón E, Wong WT, et al. Impairments in dark adaptation are associated with age-related macular degeneration severity and reticular pseudodrusen. Ophthalmology. 2015;122(10): 2053-2062.

14. Owsley C, McGwin G Jr, Clark ME, et al. Delayed rod-mediated dark adaptation is a functional biomarker for incident early age-related macular degeneration. Ophthalmology. 2016;123(2):344-351.

15. Sagong M, van Hemert J, Olmos de Koo LC, Barnett C, Sadda SR. Assessment of accuracy and precision of quantification of ultrawidefield images. Ophthalmology. 2015;122(4):864-866.

16. Grading diabetic retinopathy from stereoscopic color fundus photographs - an extension of the modified Airlie House classification. ETDRS report number 10. Early Treatment Diabetic Retinopathy Study Research Group. Ophthalmology. 1991;98(5 Suppl):786-806.

17. Csutak A, Lengyel I, Jonasson F, et al. Agreement between image grading of conventional $\left(45^{\circ}\right)$ and ultra wide-angle $\left(200^{\circ}\right)$ digital images in the macula in the Reykjavik eye study. Eye (Lond). 2010;24(10): $1568-1575$.

18. Azzolini C, Torreggiani A, Eandi C, et al. A teleconsultation network improves the efficacy of anti-VEGF therapy in retinal diseases. J Telemed Telecare. 2013;19(8):437-442.

PubMed Central and CAS, and is the official journal of The Society of Clinical Ophthalmology (SCO). The manuscript management system is completely online and includes a very quick and fair peer-review system, which is all easy to use. Visit http://www.dovepress.com/ testimonials.php to read real quotes from published authors. 